\title{
Pierre Robin Sequence
}

\author{
Noopur Gangopadhyay, M.D. ${ }^{1}$ Derick A. Mendonca, M.D., F.R.C.S. ${ }^{1}$ Albert S. Woo, M.D. ${ }^{1}$ \\ 1 Plastic and Reconstructive Surgery, Washington University School of \\ Medicine, Saint Louis, Missouri \\ Address for correspondence and reprint requests Albert S. Woo, \\ M.D., Division of Plastic and Reconstructive Surgery, Washington \\ University School of Medicine, 660 South Euclid Avenue, Campus Box \\ Semin Plast Surg 2012;26:76-82. \\ 8238, Saint Louis, MO 63110 (e-mail: WooA@wustl.edu).
}

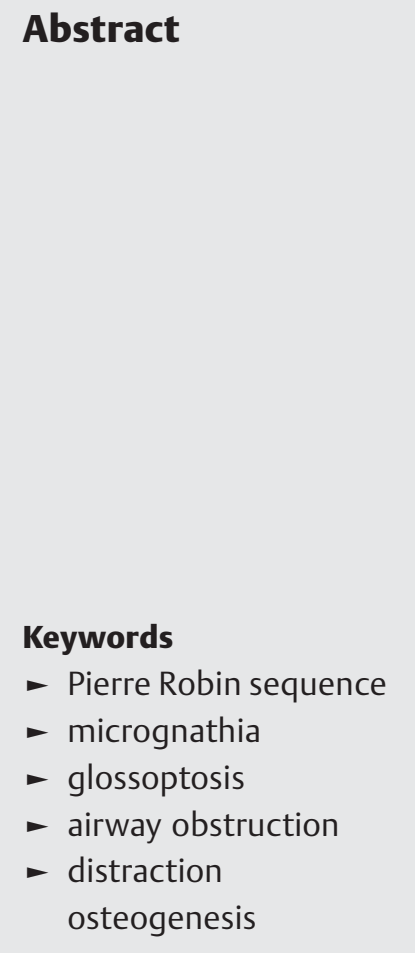

Pierre Robin sequence (PRS) is classically described as a triad of micrognathia, glossoptosis, and airway obstruction. Infants frequently present at birth with a hypoplastic mandible and difficulty breathing. The smaller mandible displaces the tongue posteriorly, resulting in obstruction of the airway. Typically, a wide U-shaped cleft palate is also associated with this phenomenon. PRS is not a syndrome in itself, but rather a sequence of disorders, with one abnormality resulting in the next. However, it is related to several other craniofacial anomalies and may appear in conjunction with a syndromic diagnosis, such as velocardiofacial and Stickler syndromes.

Infants with PRS should be evaluated by a multidisciplinary team to assess the anatomic findings, delineate the source of airway obstruction, and address airway and feeding issues. Positioning will resolve the airway obstruction in $\sim 70 \%$ of cases. In the correct position, most children will also be able to feed normally. If the infant continues to show evidence of desaturation, then placement of a nasopharyngeal tube is indicated. Early feeding via a nasogastric tube may also reduce the amount of energy needed and allow for early weight gain. A proportion of PRS infants do not respond to conservative measures and will require further intervention. Prior to considering any surgical procedure, the clinician should first rule out any sources of obstruction below the base of the tongue that would necessitate a tracheostomy. The two most common procedures for treatment, tongue-lip adhesion and distraction osteogenesis of the mandible, are discussed.

In 1923, Pierre Robin, a French stomatologist, documented a disorder which now bears his namesake. ${ }^{1,2}$ Pierre Robin sequence (PRS) was originally described as consisting of micrognathia (which he termed "mandibular hypotrophy") and glossoptosis (an abnormal posterior placement of the tongue), which result in airway obstruction and feeding difficulties. ${ }^{2}$ The small mandible is thought to be due to an inherent genetic problem or a deformational problem where intrauterine growth is restricted or mandibular positioning is altered. Rather than a syndrome, which is defined as multiple anomalies arising from a single underlying pathogenesis, it is important to note that PRS is a sequence, where multiple anomalies result from a sequential chain of malformationsone entailing the next. ${ }^{3}$ In PRS, the micrognathia leads to glossoptosis, which in turn results in airway obstruction and inability to feed.

\section{Genetic Basis}

Pierre Robin sequence occurs in $1 / 8500$ to $1 / 14,000$ births. ${ }^{4}$ This phenotype is due to several causes and can be seen in isolation or in conjunction with a syndromic presentation. Support for a genetic basis is evidenced by a high incidence of twins with PRS. Moreover, family members of PRS infants have a higher incidence of cleft lip and palate. ${ }^{5}$ Cleft palate is associated with deletions on $2 \mathrm{q}$ and $4 \mathrm{p}$, and duplications on $3 \mathrm{p}, 3 \mathrm{q}, 7 \mathrm{q}, 78 \mathrm{q}, 10 \mathrm{p}, 14 \mathrm{q}, 16 \mathrm{p}$, and 22q. Micrognathia is associated with deletions in $4 \mathrm{p}, 4 \mathrm{q}, 6 \mathrm{q}$, and $11 \mathrm{q}$, and
Issue Theme Craniofacial Surgery; Guest Editor, Edward P. Buchanan, M.D.
Copyright (c) 2012 by Thieme Medical Publishers, Inc., 333 Seventh Avenue, New York, NY 10001, USA. Tel: +1(212) 584-4662.
Dol http://dx.doi.org/ 10.1055/s-0032-1320065. ISSN 1535-2188. 


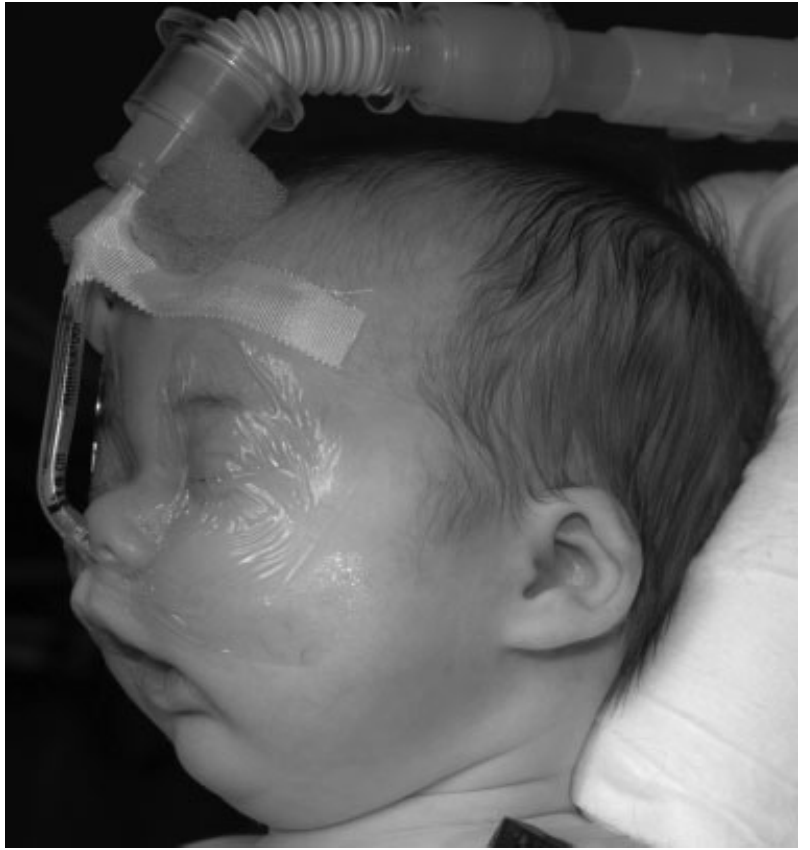

Figure 1 Microretrognathia in an infant with Pierre Robin sequence, best seen on lateral view.

duplications on $10 \mathrm{q}$ and $18 \mathrm{q} \cdot{ }^{5} \mathrm{~A}$ new study by Izumi et al evaluated two cohorts of patients clinically diagnosed with PRS using fluorescence in situ hybridization (FISH) and array comparative genomic hybridization (CGH). This study concluded that $40 \%$ of PRS was isolated and $60 \%$ were associated with another syndrome, most commonly Stickler and velocardiofacial syndromes. ${ }^{4}$ Stickler syndrome is associated with mutations in COL2A1, COL9A1, COL11A1, and COL11A2, whereas velocardiofacial syndrome arises from a microdeletion of chromosome 22q11.2. Jakobsen et al suggests that nonsyndromic PRS is associated with SOX9 and KCNJ2 dysregulation, both on chromosome 17 , based on a series of unrelated PRS patients, one of whom had a balanced translocation between chromosomes 2 and $17 .^{6}$

\section{Clinical Presentation and Diagnostic Criteria}

PRS is characterized by a classic triad of micrognathia, glossoptosis, and airway obstruction. Microretrognathia is immediately identified at birth and is a defining feature of the diagnosis (-Fig. 1). Hypoplastic mandibles are small in both the vertical and horizontal dimensions. This therefore accounts for the decrease in the anteroposterior projection of the jaw and its consequent classic retrognathic appearance. Apart from the micrognathia, Randall described the notable finding of retrogenia, or posterior displacement of the chin, to characterize the initiating anomaly in this sequence. ${ }^{3}$

Glossoptosis, defined as an abnormal posterior placement of the tongue, is the second characteristic feature of PRS. The position of the tongue is largely determined by the size and orientation of the mandible. As a smaller mandible has less anterior projection, the tongue will in turn be shifted poste- riorly. Moreover, although the tongue is typically noted to be of normal size, the hypoplastic mandible provides less volume in the oral cavity and forces the tongue to fit into a smaller space, which further serves to exacerbate the blockage of the posterior pharynx.

Airway obstruction, the completion of the triad, is a result of the abnormal positioning of the tongue, which serves to occlude the nasal and oral pharynx on inspiration. ${ }^{1,2}$ Infants with PRS may have an obstruction of the airway at the level of the tongue base, which can result in repeated oxygen desaturations, apnea, and cyanosis. ${ }^{7}$ To combat the obstructive forces, a high volume of energy is expended to continue breathing and is manifest by suprasternal retractions and the use of accessory muscles of respiration. Depending on the severity of airway obstruction, some infants may maintain their airway when awake but suffer from obstruction when asleep, especially in the supine position. ${ }^{7}$

Feeding difficulties are common as infants struggle to breathe during eating. Gastroesophageal reflux and aspiration are common sequelae of this process. The associated cleft prevents the formation of negative intraoral pressure, which is required to suck milk from the breast or bottle; the micrognathia and glossoptosis further impede mechanical sucking. ${ }^{8}$ Airway obstruction and resulting negative intrathoracic pressures have been identified as factors associated with increased gastroesophageal reflux. ${ }^{9}$ Given the poor caloric intake associated with reflux and difficulty feeding and the increased respiratory effort driving increased energy expenditure, these infants often fail to thrive and are unable to gain weight during the early postnatal period.

Pierre Robin sequence is commonly associated with a wide U-shaped cleft palate, but this is not essential to the diagnosis. ${ }^{1}$ In patients with severe micrognathia, the tongue takes up proportionately more volume in the oropharynx, resulting in glossoptosis in the setting of a small mandible. ${ }^{10}$ During embryonic development, vertically oriented palatal shelves are mobilized to a horizontal position during the eighth week of development. Numerous theories have been suggested to account for the very common finding of cleft palate in PRS. One notable theory that has been proposed is the relatively large tongue may serve as a physical barrier to this movement, resulting in a cleft palate. ${ }^{10}$ Despite its absence in the classic triad, cleft palate remains the most common associated abnormality in PRS. Moreover, $80 \%$ of infants diagnosed with PRS have other associated anomalies. ${ }^{7}$

\section{Associated Syndromes}

Pierre Robin sequence is related to several other craniofacial anomalies and may appear in conjunction with the findings characteristic of several different syndromes. Stickler syndrome, an autosomal dominant condition, is characterized by a short mandibular ramus, antegonial notching of the mandibular body, myopia, and joint problems. ${ }^{1}$ Velocardiofacial syndrome is characterized by a retrognathic mandible, palatal abnormalities, hypotonia, impaired thymus development, and cardiac malformations. ${ }^{3,4}$ Craniofacial microsomia (or oculoauriculovertebral spectrum) results in anomalies of the 
external and middle ear, mandible, zygoma, maxilla, temporal bone, facial muscles, and palate. ${ }^{2}$ Treacher Collins syndrome is notable for dysplasia affecting the zygoma, temple, ear, and mandible. It is further characterized by downslanting palpebral fissures, lower lid colobomas, facial bone hypoplasia, malformation of the external ear, macrostomia, and a high-arching palate. Some have likened this to Tessier 6, 7, and 8 facial clefts. ${ }^{1,10}$

\section{Assessment}

Infants with PRS should be evaluated in a team setting to assess the anatomic findings, delineate the source of airway obstruction, and address feeding issues to maximize growth and minimize obstruction. ${ }^{2}$ A multidisciplinary approach is ideally suited for this task, consisting of specialists from plastic and reconstructive surgery, pediatric otolaryngology, pediatric pulmonology, speech pathology, nursing, pediatric anesthesia, and neonatology. ${ }^{8} \mathrm{~A}$ geneticist should also be involved if there is any concern for an underlying syndrome or genetic basis for diagnosis. Patients suspected of having Stickler syndrome should have an ophthalmology examination.

Glossoptosis may result in upper airway obstruction in PRS, but these patients can have several other reasons for airway compromise due to syndromic causes. Skull-base anomalies as in Stickler or Treacher Collins syndromes, nasal constriction seen in Treacher Collins or velocardiofacial syndrome, and central sleep apnea secondary to central nervous system depression and pharyngeal hypotonia as in velocardiofacial syndrome can all result in airway obstruction. ${ }^{7}$ Furthermore, PRS infants may also have a short or collapsing epiglottis, laryngomalacia, and segments of tracheal stenosis all exacerbating airway obstruction. ${ }^{11,12}$

Evaluation of the airway in PRS is critical for decision making for treatment. Helpful studies include sleep monitoring for episodes of spontaneous oxygen desaturation, desaturations during feeding, during sleep, and during phonation.,8 Nasoendoscopy and bronchoscopy are invaluable adjuncts to determining the site of airway obstruction, as there may be more sources of airway compromise than the tongue base itself, such as in laryngomalacia, tracheomalacia, or other subglottic obstructions. ${ }^{11,12}$ Part of the assessment will include evaluation of patients in different positions and how well positioning resolves upper airway obstruction. ${ }^{8,11}$

\section{Management}

\section{Nonsurgical Management}

Prone or lateral positioning will solve the airway obstruction in $\sim 70 \%$ of cases of PRS. ${ }^{2,8}$ With appropriate positions, many of these children will also be able to feed normally and no further treatment is necessary. If the baby continues to show evidence of desaturation, then placement of a nasopharyngeal (NP) tube is indicated, where the aim of the tube placement is to bypass the site of upper airway obstruction (-Fig. 2). Chang et al have discussed the technique of creating individualized NP tubes from endotracheal tubes, with the

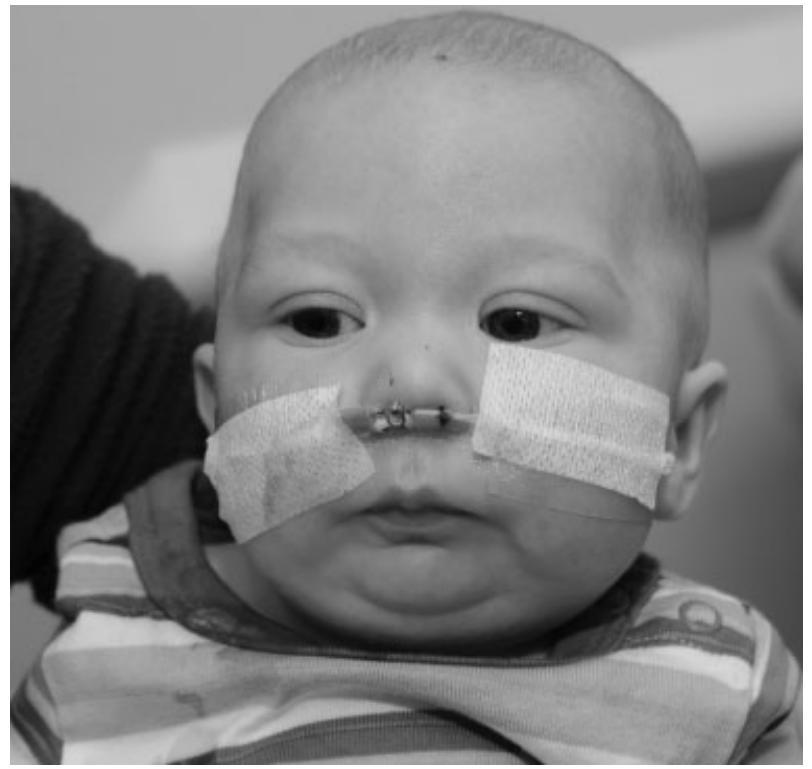

Figure 2 Nasopharyngeal airway used in treatment of Pierre Robin sequence.

diameter and length chosen according to the infant's weight. ${ }^{13,14}$ They report the successful and safe usage of a modified NP airway over the traditional NP airway in relieving airway obstruction and also reducing the need for surgical intervention. The modified NP airway has less dead space due to reduced bulk and allows for simultaneous use of nasal prongs for supplemental oxygen. ${ }^{13}$ Maintenance of the modified NP airway along with replacement and cleaning can be done by the nursing staff and taught to the parents, allowing for home management of these babies. ${ }^{14}$ Supportive airways are typically needed for 2 to 4 months for respiratory support.

Attention to feeding is the second most important factor in treating babies with PRS. ${ }^{14-16}$ Infants who are under no respiratory distress may still demonstrate difficulty with the active process of feeding, especially with an associated cleft palate. Early feeding via nasogastric tube reduces the amount of energy needed and allows for early weight gain. ${ }^{15}$ A weight gain of 20 to $30 \mathrm{~g} / \mathrm{d}$ is considered satisfactory. ${ }^{16}$ Recently, it has been reported that severe PRS babies can have low urinary sodium. ${ }^{17}$ Sodium supplementation in such babies with low urinary sodium improved their weight gain and reversed the tendency to failure to thrive. ${ }^{17}$ However, it should be noted that nonoperative treatment is more likely to succeed with nonsyndromic patients than with syndromic patients. ${ }^{14}$

It is widely believed that there is a subset of PRS infants that do not respond to conservative measures and will require further intervention. Temporizing measures such as supplemental oxygen, nasopharyngeal tubes, laryngeal masks, and prolonged intubation are inadequate in infants with severe respiratory distress. The most common methods for surgical management of airway obstruction include tongue-lip adhesion, distraction osteogenesis, and tracheostomy. 


\section{Tongue-Lip Adhesion}

Tongue-lip adhesion (TLA) was first described by Shukowsky in 1911 and popularized by Douglas in the mid-20th century. ${ }^{18}$ The procedure serves to correct the problem of glossoptosis by pulling the base of the tongue forward and suturing it to the lower lip. Once healed, this mucosal attachment serves to tether the tongue anteriorly until the infant develops a more stable airway with growth. TLA can only be performed on infants who have not developed any lower teeth, as they could otherwise bite through the repair inadvertently. After adequate growth has occurred, the TLA must be released with a second procedure.

TLA is well described in the plastic surgery literature and several modifications have been published in recent years. A traditional method is described here ( $\mathbf{- F i g . ~ 3 ) . ~}{ }^{19}$ Prior to intubation, a direct laryngoscopy and bronchoscopy must be performed to exclude supraglottic and subglottic airway

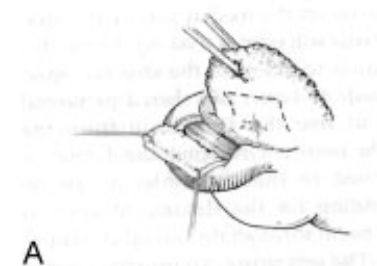

A

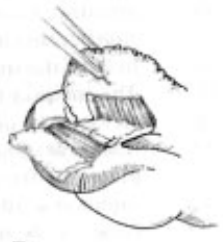

B

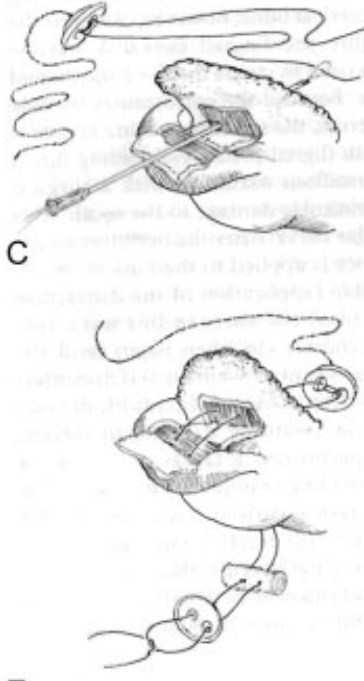

E

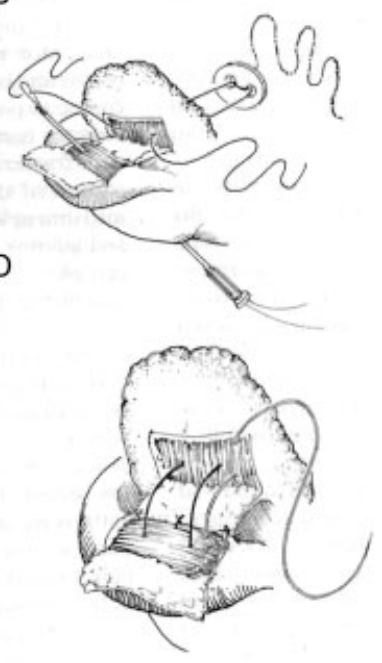

F

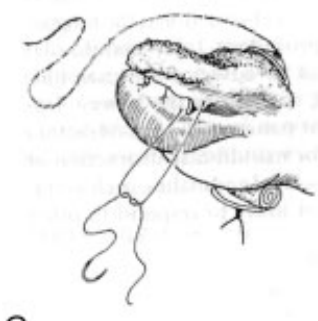

G

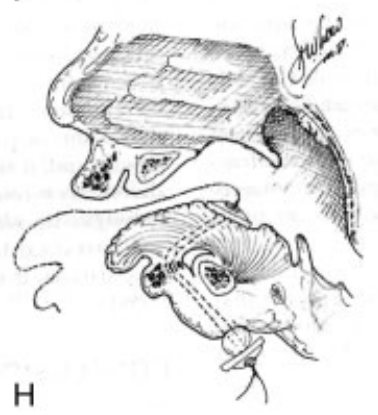

$\mathrm{H}$

Figure 3 A schematic of tongue-lip adhesion illustrating overlapping mucosal-based flaps and the posterior tongue/inferior chin buttons. (Reprinted with permission from Bartlett SP, Losee JE, Baker SB. Reconstruction: craniofacial syndromes. In: Mathes SJ, ed. Plastic Surgery. Vol. 4. Philadelphia, PA: Saunders Elsevier, 2006: 516.) anomalies. The infant is then intubated and placed in a supine position. A 3-0 polypropylene suture is passed transversely through the tongue as a traction suture and opposing mirrorimage rectangular mucosal flaps are designed on the lower lip and ventral tongue. ${ }^{19}$ Local anesthetic with epinephrine is infiltrated along the incisions. The genioglossus is released with a Cottle elevator through the ventral tongue incision and elevated from the lingual mandible. ${ }^{18}$ A 4-0 polypropylene suture is passed around the mandible and brought through lingual muscle, and then tied across the alveolar ridge in a buried fashion as the tongue is pulled forward with the traction stitch. ${ }^{18}$ The mucosal flaps are closed with 4-0 interrupted chromics and the musculature is closed with 4-0 Vicryl. The tongue traction stitch is secured to the lower lip and chin with adhesive strips and can be pulled forward if needed postoperatively. Two sutures pass vertically through the TLA and connect to a button from the posterior tongue to a button on the inferior external chin. ${ }^{19}$ These buttons and sutures relieve tension on the tongue-lip flap as the wound heals. Critical features of this procedure as described by Rogers et al include the genioglossus release and the buried circum-mandibular suture. ${ }^{18} \mathrm{~A}$ postoperative picture is shown in - Fig. 4. The tongue traction suture and buttons are removed at 7 to 12 days postoperatively. ${ }^{19,20}$ Between the ages of 9 months to 1 year, the tongue is released. ${ }^{20}$ Postoperative complications include partial or total dehiscence of the repair, reoperation, soft tissue infection or abscess, unacceptable base of tongue scarring, and conversion to tracheostomy. ${ }^{20}$

Most authors conclude that TLA is a suitable option for infants whose airway obstruction is isolated to the base of

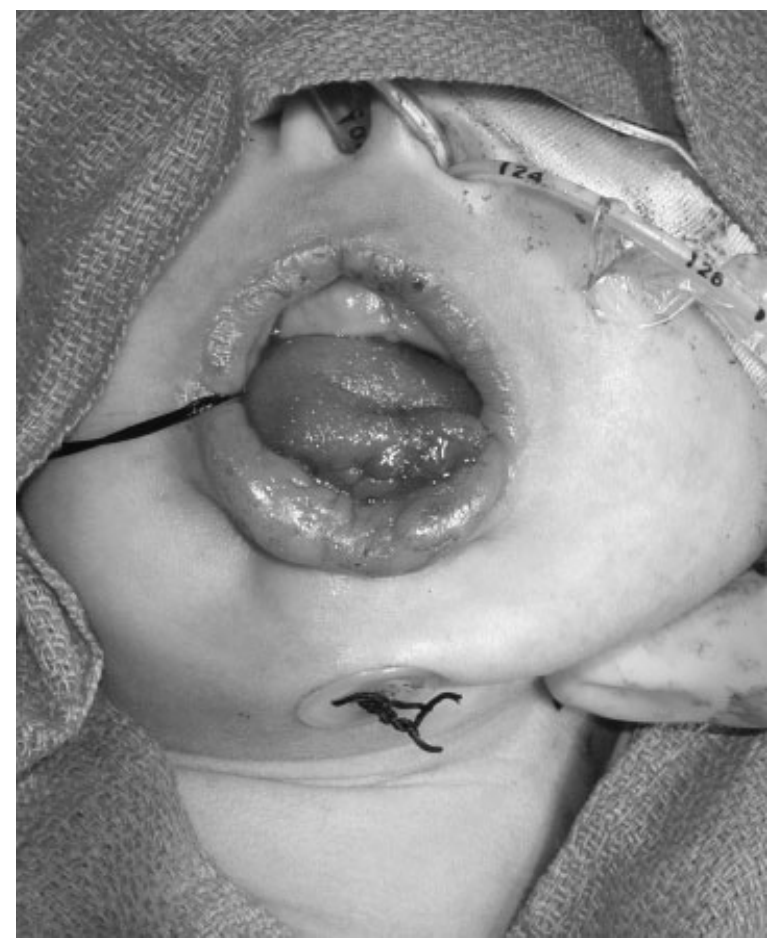

Figure 4 Postoperative view of patient after tongue-lip adhesion for airway obstruction. (Courtesy of Dr. Alex Kane.) 
tongue and who continue to desaturate with prone positioning. Numerous studies have evaluated the efficacy of TLA in protecting the airway in children with PRS. Kirschner et al reported the outcomes of 29 patients undergoing TLA, finding $83.3 \%$ success in relieving airway obstruction and a reduction in NG tube feedings from $93.1 \%$ preoperatively to $72.4 \%$ postoperatively, with $62 \%$ of infants being weaned from all NG tube feeds within 6 months. ${ }^{21}$ Evaluation of PRS infants for obstructive sleep apnea before and after TLA show that TLA decreases the obstructive apnea hypopnea index (number of obstructive apneas and hypopneas divided by total sleep time) by decreasing the number of events per hour, decreases the peak end-tidal $\mathrm{pCO}_{2}$ measurement and improves the oxygen saturation. ${ }^{22}$ Although obstructive sleep apnea improves in most cases after TLA, complete resolution of symptoms is found in only $38 \%$ of patients. ${ }^{22}$ Denny, Amm, and Schaefer reviewed their experience with TLA and concluded that though the initial success rate was high for correction of airway obstruction, most patients with severe respiratory distress required a secondary intervention within the first year of life such as mandibular distraction and tracheostomy for airway management and gastrostomy tube placement for nutritional support. ${ }^{23}$

\section{Distraction Osteogenesis of the Mandible}

Distraction osteogenesis (DO) of the mandible, first described in 1989 by McCarthy, has become popular as the definitive technique to address the issues associated with PRS by relieving airway obstruction, improving facial cosmesis, and correcting malocclusion. ${ }^{24}$ The process of mandibular distraction lengthens the jaw in a forward direction, and also indirectly pulls the tongue base anteriorly. As the mandible is projected forward, the tongue is also pulled anteriorly through its muscular attachments on the lingual surface of the mandible. Thus, this technique reverses the sequence of PRS by correcting the micrognathia, which in turn improves the glossoptosis, thereby relieving the obstruction of the airway.

Mandibular distraction is reserved for patients with tongue-base airway obstruction who experience failure with positioning and conservative measures. Infants who are candidates for acute mandibular DO in the neonatal period are those that would otherwise undergo tracheotomy for severe airway distress, as evidenced by retractions, stridor, hypoxia, hypercarbia, and poor oral intake resulting in weight loss. ${ }^{25}$ On endoscopic exam, these infants have tongue base obstruction of the hypopharynx and compression supraglottically due to a microretrognathic mandible; a jaw thrust maneuver will improve symptoms and mimics the ultimate result of DO. ${ }^{25}$ A preoperative three-dimensional computed tomography (CT) scan is useful for surgical planning to assess the location of proposed osteotomies in relation to tooth buds and nerve roots.

A significant degree of variability is present among surgeons in regards to technique for distraction. Oblique osteotomies at the angle of the mandible remain the most common approach for distraction. Multiple authors have also advocated an inverted-L approach to minimize injury to the tooth buds, which are present along the region of the internal angle. ${ }^{26}$
Denny and Amm have argued for a horizontal osteotomy in the mandibular ramus and recommend distracting in a vertical vector to avoiding damaging the tooth roots. ${ }^{27}$ Molina has suggested a more oblique vector and favors an external distractor that allows multivector control of the generate. ${ }^{28} \mathrm{Mc}-$ Carthy, on the other hand, has advocated a more horizontal vector, noting that the deficiency in PRS is horizontal rather than vertical (as in craniofacial microsomia). ${ }^{29}$

Our senior author's method for internal mandibular distraction osteogenesis is as follows. Under general anesthesia, the patient is placed in a supine position on the operating room table. As previously mentioned, prior to any surgical intervention a direct laryngoscopy and bronchoscopy are performed. An otolaryngologist confirms the presence of upper airway obstruction and rules out subglottic anomalies that would not be corrected with a distraction procedure. Following nasotracheal intubation, a standard Risdon incision is made through the skin overlying the angle of the mandible. Once the platysma has been incised, a nerve stimulator is used to avoid injury to the marginal mandibular branch of the facial nerve during dissection. The periosteum is incised over the angle of the mandible and the lingual and buccal cortices are exposed. A 270-degree osteotomy is then made in an oblique fashion along the mandibular angle. ${ }^{28}$ This technique entails initial superficial osteotomy of the outer cortex of the mandible with a reciprocating saw, followed by osteotomies superiorly and inferiorly, leaving the middle third of the mandible unharmed to preserve the patency of the inferior alveolar nerve. (The course of this nerve can be identified preoperatively with a high-resolution CT scan.) The osteotomy is completed by placing a straight osteotome in one of the osteotomy sites and gently rotating the instrument until the lingual surface fractures from the tension (-Fig. 5). Once this occurs, the inferior alveolar nerve can be visualized to be intact. An internal (or external) distraction device is then placed. When an internal distractor is utilized, the distraction arm can exit the skin through a separate incision underneath the ear lobule (-Figs. 6 and 7). The absence of obstruction to distraction is confirmed with 2 to 3 pin rotations. The bone segments are returned to their initial positions prior to beginning the distraction protocol postoperatively. The platysma and skin are then closed with 5-0 Monocryl. This procedure is performed bilaterally.

Surgeons vary in the length of the latency period before distraction is initiated. This period can range from 24 hours to 7 days, though most surgeons wait 2 to 3 days before activating distraction. ${ }^{25}$ It is the senior author's practice to begin neonatal distraction within 24 hours of the procedure, whereas older individuals undergo a 5-day latency period. Distraction rates range from 0.5 to $2 \mathrm{~mm}$ per day spread out in two to three sessions per day. ${ }^{24,27}$ Our institution distracts neonates at $2 \mathrm{~mm} /$ day and older subjects at $1 \mathrm{~mm} /$ day. This is followed by a 4- to 6 -week consolidation phase. Complications of DO include pin site infections, inferior alveolar neurapraxia, injury to the tooth roots, unacceptable scarring, resorption/ankylosis at the temporomandibular joint, malunion, and failure of distraction due to incomplete osteotomies, early consolidation, or device failure. ${ }^{25,27}$ 


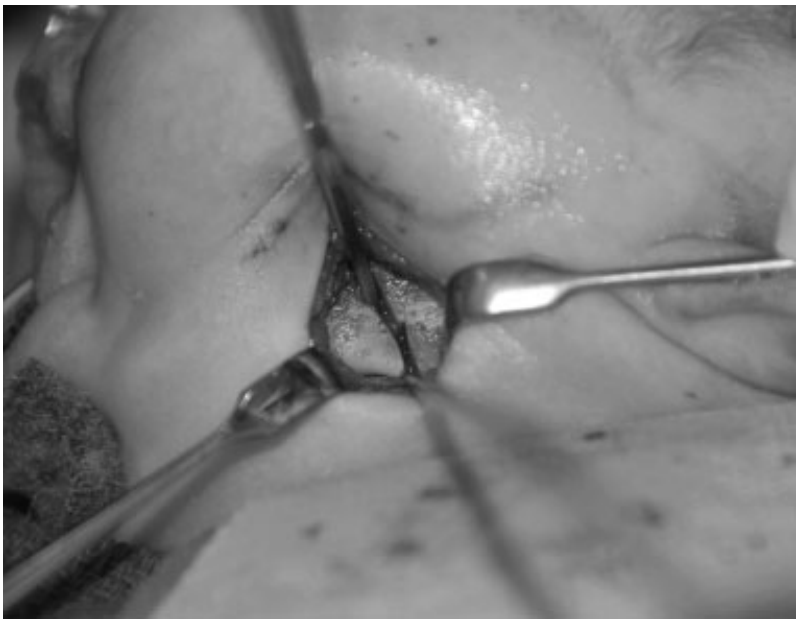

Figure 5 A 270-degree osteotomy for distraction osteogenesis. Note that the anterior cortex has been osteotomized. A straight osteotome is placed in the bone and canted to complete the fracture along the lingual cortex. The inferior alveolar nerve can be partially visualized in the midportion of the mandible.

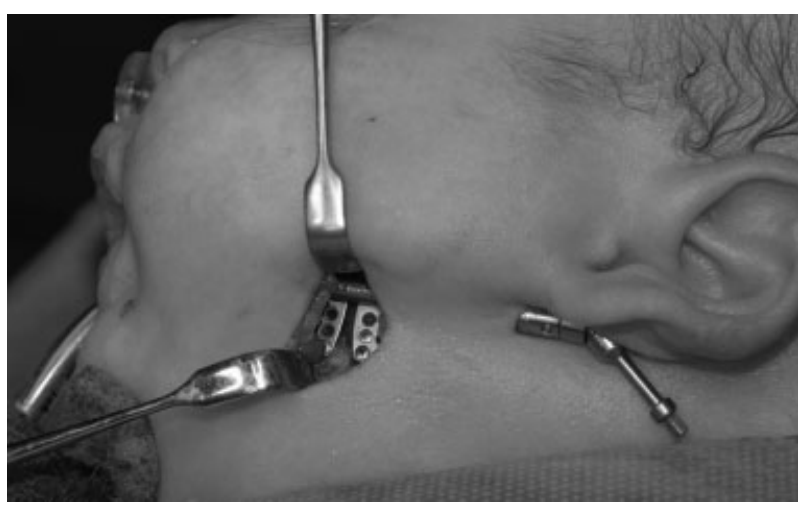

Figure 6 Internal univector distractor placed following osteotomy. The distraction arm exits the skin via an incision inferior to the ear lobule.

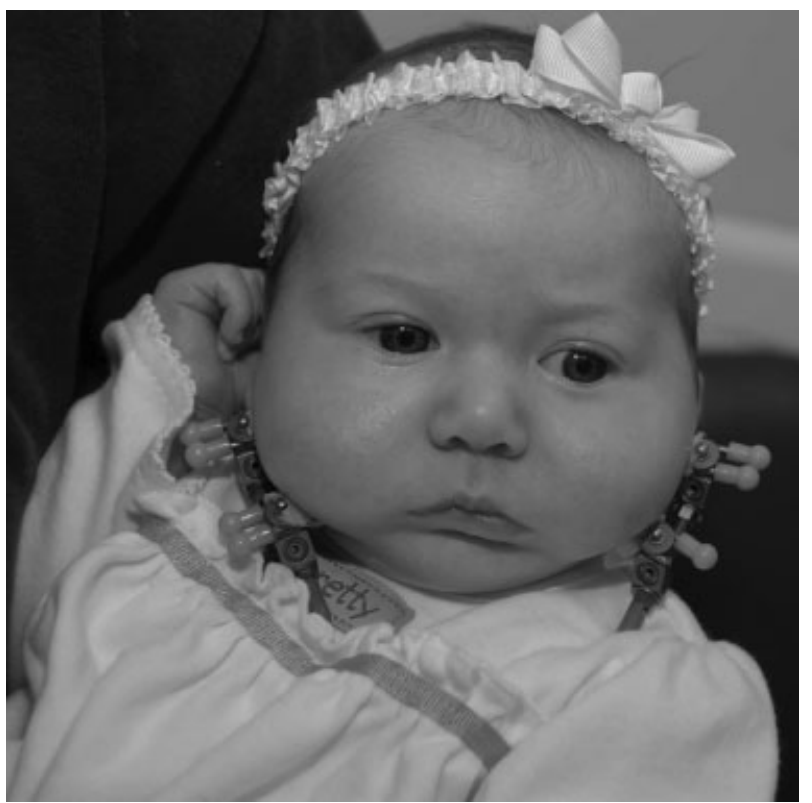

Figure 7 External distraction multivector distractor.
The major advantage of DO is the avoidance of tracheotomy for airway management. Moreover, in several reported series, those patients who underwent tracheotomy prior to DO were able to be successfully decannulated after DO was complete. $^{24}$ The main physiological sequelae of PRS is an inability to effectively feed and/or breathe because of airway obstruction. Ortiz-Monasterio observed that $83 \%$ of patients with PRS demonstrated gastroesophageal reflux disease at presentation and all patients exhibited resolution of the reflux with adequate airway control. ${ }^{30}$ At our own institution, neonates receiving mandibular distraction osteogenesis have been shown to have improved feeding outcomes. ${ }^{15}$ Infants gain weight faster and are more likely to be discharged on $100 \%$ oral feeds as opposed to NG tube feeds in those that did not have distraction. These results were independent of the syndromic status of the patients. ${ }^{15}$

\section{Decision Making in the Treatment of Pierre Robin Sequence}

The treatment of infants with PRS remains controversial and differs among institutions. Our approach to the infant with PRS begins with attempts at prone positioning to eliminate the effect of gravity on the base of the tongue. Continuous pulse oximetry is performed on all patients. If positioning alone is not successful, adjunctive measures such as supplemental oxygen and modified nasopharyngeal tubes are used to bypass the tongue base obstruction. We find that polysomnography can be a useful tool to document the obstructive apnea-hypopnea index and confirm the absence of unrecognized obstructive or central sleep apnea.

Another key feature of our treatment focuses on feeding and nutritional support. All patients are evaluated closely in regards to their ability to feed by qualified speech therapists who specialize in feeding. If necessary, early nasogastric feeding is initiated to supplement oral feeds and improve weight gain. Most infants will be successfully managed with conservative measures alone. If these measures fail to relieve obstruction as evidenced by inadequate results on sleep studies and poor weight gain, surgical options are considered.

Prior to any surgical intervention, we confirm the absence of obstruction below the level of the tongue base with direct laryngoscopy and bronchoscopy performed by a pediatric otolaryngologist. Though numerous algorithms are described in the literature in regards to surgical decision making, no uniform consensus currently exists. The clear benefits of one surgical technique versus another have not clearly been determined and neither procedure can be expected to provide normal occlusion for individuals at the time of skeletal maturity. At our institution, the risks and benefits of both tongue-lip adhesion and mandibular distraction osteogenesis are discussed with the family and a multidisciplinary team approach is used to generate the final surgical treatment plan based on the estimated duration of respiratory support, failure of nonoperative management, and the surgeon's clinical judgment. Tracheostomy remains the gold standard for definitive airway protection and is the only option for infants with an associated subglottic obstruction and tracheomalacia. 


\section{References}

1 Sommerlad B. Cleft palate. In: Guyuron B, Eriksson E, Persing J, eds. Plastic Surgery Indications and Practice. Philadelphia, PA: Saunders Elsevier; 2009:508-509

2 Mackay DR. Controversies in the diagnosis and management of the Robin sequence. J Craniofac Surg 2011;22(2):415-420

3 Hunt JA, Hobar PC. Common craniofacial anomalies: the facial dysostoses. Plast Reconstr Surg 2002;110(7):1714-1725, quiz 1726, discussion 1727-1728

4 Izumi K, Konczal LL, Mitchell AL, et al. Underlying genetic diagnosis of Pierre Robin sequence: retrospective chart review at two children's hospitals and a systematic literature review. J Pediatr 2012; 160(4):645-650, e2

5 Jakobsen LP, Knudsen MA, Lespinasse J, et al. The genetic basis of the Pierre Robin sequence. Cleft Palate Craniofac J 2006; 43(2):155-159

6 Jakobsen LP, Ullmann R, Christensen SB, et al. Pierre Robin sequence may be caused by dysregulation of SOX9 and KCNJ2. J Med Genet 2007;44(6):381-386

7 Shprintzen RJ. The implications of the diagnosis of Robin sequence. Cleft Palate Craniofac J 1992;29(3):205-209

8 Marcellus L. The infant with Pierre Robin sequence: review and implications for nursing practice. J Pediatr Nurs 2001;16(1):23-34

9 Marques IL, Monteiro LC, de Souza L, Bettiol H, Sassaki CH, de Assumpção Costa R. Gastroesophageal reflux in severe cases of Robin sequence treated with nasopharyngeal intubation. Cleft Palate Craniofac J 2009;46(4):448-453

10 Gosain AK, Nacamuli R. Embryology of the head and neck. In: Thorne CH, Beasley RW, Aston SJ, et al, eds. Grabb \& Smith's Plastic Surgery. 6th ed. Philadelphia, PA: Lippincott Williams \& Wilkins; 1997:179-190

11 Cheng AT, Corke M, Loughran-Fowlds A, Birman C, Hayward P, Waters KA. Distraction osteogenesis and glossopexy for Robin sequence with airway obstruction. ANZ J Surg 2011;81(5): 320-325

12 Cruz MJ, Kerschner JE, Beste DJ, Conley SF. Pierre Robin sequences: secondary respiratory difficulties and intrinsic feeding abnormalities. Laryngoscope 1999;109(10):1632-1636

13 Chang AB, Masters IB, Williams GR, Harris M, O'Neil MC. A modified nasopharyngeal tube to relieve high upper airway obstruction. Pediatr Pulmonol 2000;29(4):299-306

14 Anderson KD, Cole A, Chuo CB, Slator R. Home management of upper airway obstruction in Pierre Robin sequence using a nasopharyngeal airway. Cleft Palate Craniofac J 2007;44(3):269-273

15 Al-Samkari HT, Kane AA, Molter DW, Vachharajani A. Neonatal outcomes of Pierre Robin sequence: an institutional experience. Clin Pediatr (Phila) 2010;49(12):1117-1122
16 Wagener S, Rayatt SS, Tatman AJ, Gornall P, Slator R. Management of infants with Pierre Robin sequence. Cleft Palate Craniofac J 2003;40(2):180-185

17 Skillman J, Cole A, Slator R. Sodium supplementation in neonates with Pierre Robin sequence significantly improves weight gain if urinary sodium is low. Cleft Palate Craniofac J 2012;49(1):39-43

18 Rogers GF, Murthy AS, LaBrie RA, Mulliken JB. The GILLS score: part I. Patient selection for tongue-lip adhesion in Robin sequence. Plast Reconstr Surg 2011;128(1):243-251

19 Bartlett SP, Losee JE, Baker SB. Reconstruction: craniofacial syndromes. In: Mathes SJ, ed. Plastic Surgery. Vol. 4. Philadelphia, PA: Saunders Elsevier; 2006:514-516

20 Bijnen CL, Don Griot PJW, Mulder WJ, Haumann TJ, Van Hagen AJ Tongue-lip adhesion in the treatment of Pierre Robin sequence. J Craniofac Surg 2009;20(2):315-320

21 Kirschner RE, Low DW, Randall P, et al. Surgical airway management in Pierre Robin sequence: is there a role for tongue-lip adhesion? Cleft Palate Craniofac J 2003;40(1):13-18

22 Sedaghat AR, Anderson IC, McGinley BM, Rossberg MI, Redett RJ Ishman SL. Characterization of obstructive sleep apnea before and after tongue-lip adhesion in children with micrognathia. Cleft Palate Craniofac J 2012;49(1):21-26

23 Denny AD, Amm CA, Schaefer RB. Outcomes of tongue-lip adhesion for neonatal respiratory distress caused by Pierre Robin sequence. J Craniofac Surg 2004;15(5):819-823

24 Boston M, Rutter MJ. Current airway management in craniofacial anomalies. Curr Opin Otolaryngol Head Neck Surg 2003; 11(6):428-432

25 Fritz MA, Sidman JD. Distraction osteogenesis of the mandible. Curr Opin Otolaryngol Head Neck Surg 2004;12(6):513-518

26 Kobayashi A, Yoshimasu H, Kobayashi J, Amagasa T. Neurosensory alteration in the lower lip and chin area after orthognathic surgery: bilateral sagittal split osteotomy versus inverted $L$ ramus osteotomy. J Oral Maxillofac Surg 2006;64(5):778-784

27 Denny A, Amm C. New technique for airway correction in neonates with severe Pierre Robin sequence. J Pediatr 2005; 147(1):97-101

28 Molina F, Ortiz Monasterio F. Mandibular elongation and remodeling by distraction: a farewell to major osteotomies. Plast Reconstr Surg 1995;96(4):825-840, discussion 841-842

29 McCarthy JG, Schreiber J, Karp N, Thorne CH, Grayson BH. Lengthening the human mandible by gradual distraction. Plast Reconstr Surg 1992;89(1):1-8, discussion 9-10

30 Monasterio FO, Molina F, Berlanga F, et al. Swallowing disorders in Pierre Robin sequence: its correction by distraction. J Craniofac Surg 2004;15(6):934-941 